$11-10-2008$

\title{
Spectral Time Moment Analysis of Microgel Deswelling: Effect of the Heating Rate
}

\author{
Kiril A. Streletzky \\ Cleveland State University, K.STRELETZKY@csuohio.edu \\ John T. McKenna \\ Cleveland State University
}

Follow this and additional works at: https://engagedscholarship.csuohio.edu/sciphysics_facpub

Part of the Physics Commons

How does access to this work benefit you? Let us know!

Publisher's Statement

This is the accepted version of the following article: Kiril A. Streletzky and John T. Mckenna, "Spectral time moment analysis of microgel deswelling. Effect of the heating rate," Journal of Polymer Science Part B: Polymer Physics 46 (24), 2792-2802 (2008). , which has been published in final form at http://onlinelibrary.wiley.com/doi/10.1002/polb.21615/full

\section{Repository Citation}

Streletzky, Kiril A. and McKenna, John T., "Spectral Time Moment Analysis of Microgel Deswelling: Effect of the Heating Rate" (2008). Physics Faculty Publications. 264.

https://engagedscholarship.csuohio.edu/sciphysics_facpub/264

This Article is brought to you for free and open access by the Physics Department at EngagedScholarship@CSU. It has been accepted for inclusion in Physics Faculty Publications by an authorized administrator of EngagedScholarship@CSU. For more information, please contact library.es@csuohio.edu. 


\title{
THERMAL FLUCTUATIONS IN SYSTEMS WITH CONTINUOUS SYMMETRY: BROWNIAN MOTION AND LÉVY-FLIGHT DESCRIPTION
}

\author{
U. ZÜRCHER
}

\begin{abstract}
We investigate relaxation and thermal fluctuations in systems with continuous symmetry in arbitrary spatial dimensions. For the scalar order parameter $\zeta(\mathbf{r}, t)$ with $\mathbf{r} \in \mathcal{R}^{d}$, the deterministic relaxation is caused by hydrodynamic modes $\eta \partial \zeta(\mathbf{r}, t) / \partial t=K \nabla^{2} \zeta(\mathbf{r}, t)$. For a finite volume $V$, we expand the scalar field in a discrete Fourier series and then we study the behavior in the limit $V \rightarrow \infty$. We find that the second moment is well defined for dimensions $d \geq 3$, while it diverges for $d=1,2$. Furthermore, we show that for $d<4$, the decay of the scalar field does not define an "effective" relaxation time. For dimensions $d<4$, these two properties suggest scale-invariant properties of the scalar field in the limit $V \rightarrow \infty$. We show that thermal fluctuations are described by fractional Brownian motion for $d \leq 3$ and by ordinary Brownian motion for $d \geq 4$. The spectral density of the stochastic force follows $1 / f$ for $d=1$ and $d=2$, $1 / \sqrt{f}$ for $d=3$, and "white noise," $f^{0}$ for $d \geq 4$. We find explicit representation of the equilibrium distribution of the conserved scalar field. For $d \geq 4$ it is a Gaussian distribution, while for $d=1$ and $d=2$, it is the Cauchy distribution.
\end{abstract}




\section{INTRODUCTION}

In this paper, we re-examine the relaxation of a scalar field $\zeta(\mathbf{r}, t)$ in $d$-dimensional space $\mathbf{r} \in \mathcal{R}^{d}$, whose total "charge" is conserved,

$$
\int_{\mathcal{R}^{d}} \zeta(\mathbf{r}, t) d \mathbf{r}=\text { const. }
$$

The relaxation of the conserved scalar field is given by a continuity equation $\eta \partial \zeta(\mathbf{r}, t) / \partial t+\nabla \cdot \mathbf{j}=\mathbf{0}$, where the flux $\mathbf{j}=\mathbf{j}[\zeta(\mathbf{r}, t)]$ has no sources or sinks. In many cases, a gradient flux is realized, i.e., $\mathbf{j}=-K \nabla \zeta(\mathbf{r}, t)$, and we arrive at the relaxation equation,

$$
\eta \frac{\partial \zeta(\mathbf{r}, t)}{\partial t}=K \nabla^{2} \zeta(\mathbf{r}, t),
$$

where $\nabla^{2}$ is the $d$-dimensional Laplacian and where $\eta$ and $K$ have units of time and the square of a length, respectively, $[\eta]=s$ and $[K]=m^{2}$.

Equation (1.2) is encountered in dynamic theories of phase transitions ${ }^{1}$; studies of "self-organized criticality" 2,3 ; relaxation of the height of granular aggregates ${ }^{4}$; relaxation processes in soft condensed matter systems ${ }^{5-7}$; and others. We refer to the above cited references for a careful discussion of how Eq. (1.2) arises in the respective physical situations.

Because Eq. (1.2) is linear, it is readily solved using Fourier transform. We define the scalar field on a $d$-dimensional lattice so that the $k$ th component of the coordinate vector is given by $[\mathbf{r}]_{k}=n_{k} a$ with $0 \leq n_{k} \leq L / a$ and $a$ being the lattice spacing. The volume follows as $V=L^{d}$. The short- and long-wavelength cutoff $a$ and $L$ introduce the upper and lower cutoff for wavevectors, respectively, $2 \pi / L \leq|\mathbf{q}| \leq 2 \pi / a$. We thus have:

$$
\zeta(\mathbf{r}, t)=V^{-1} \sum_{\mathbf{q}} \dot{\zeta}(\mathbf{q}, t) e^{i \mathbf{q} \cdot \mathbf{r}} .
$$

We find the relaxation equation for the Fourier components, $d \dot{\zeta}(\mathbf{q}, t) / d t=-\tau_{\mathbf{q}}^{-1} \dot{\zeta}(\mathbf{q}, t)$, where the relaxation times are given by $\tau_{\mathbf{q}}=\eta / K q^{2}$. That is, long wavelength modes decay arbitrarily slowly, i.e., $\tau_{\mathbf{q}} \rightarrow \infty$ for $|\mathbf{q}| \rightarrow \mathbf{0}$.

The deterministic relaxation, [cf. Eq. (1.2)], can be written in Ginzburg-Landau form, ${ }^{8}$

$$
\eta \frac{\partial \zeta(\mathbf{r})}{\partial t}=-\frac{\delta \mathcal{F}[\zeta(\mathbf{r})]}{\delta \zeta(\mathbf{r}, t)},
$$

where $\delta / \delta \zeta(\mathbf{r}, t)$ is the functional derivative and its free energy is given by $\mathcal{F}=\frac{K}{2} \int d \mathbf{r}(\nabla \zeta(\mathbf{r}))^{2}$. In
Fourier representation, we have:

$$
\mathcal{F}[\dot{\zeta}(\mathbf{q})]=\frac{K}{2} \sum_{\mathbf{q}} q^{2}|\tilde{\zeta}(\mathbf{q})|^{2} .
$$

To define thermal equilibrium properties, we assume that the system is in contact with a thermal reservoir at temperature $T$. After a long time, the system approaches a stationary state in which the scalar field is characterized by the canonical distri. bution, $\rho_{\text {eq }}[\zeta(\mathbf{r})]=Z^{-1} \exp (-\mathcal{F}[\zeta(\mathbf{r})] / T)$. Here, we use units such that $k_{B}=1$, and the normalization constant is the partition function $Z$. From Eq. (1.5) we have,

$$
\rho_{e q}[\dot{\zeta}(\mathbf{q})]=Z^{-1} \prod_{\mathbf{q}} \exp \left(-\frac{K}{2 T} q^{2}|\bar{\zeta}(\mathbf{q})|^{2}\right),
$$

so that for each Fourier mode, the distribution is a Gaussian and equilibrium properties are determined by their first and second moments,

$$
\begin{aligned}
\langle\bar{\zeta}(\mathbf{q})\rangle & =0, \\
\left\langle|\bar{\zeta}(\mathbf{q})|^{2}\right\rangle & =\frac{T}{K q^{2}} .
\end{aligned}
$$

We observe that long-wavelength modes have large variances.

Because we consider the linear relaxation of the conserved scalar field, its properties are determined by the equilibrium correlation function. $C_{e q}(\mathbf{r}, t)=\left\langle\zeta\left(\mathbf{r}+\mathbf{r}_{0}, t+t_{0}\right) \zeta\left(\mathbf{r}_{0}, t_{0}\right)\right\rangle_{e q}$. In Fourier representation, we have $C_{e q}(\mathbf{r}, t)=V^{-1} \sum_{q}$ $\left\langle|\dot{\zeta}(\mathbf{q})|^{2}\right\rangle_{e q} e^{\mathbf{i q} \cdot \mathbf{r}} e^{-t / \tau_{\mathbf{q}}}$, or, for $\mathbf{r}=\mathbf{0}$,

$$
C_{e q}(t)=V^{-1} \sum_{\mathbf{q}} \frac{T}{K q^{2}} \exp \left(-\frac{K q^{2}}{\eta} t\right),
$$

where we have inserted Eq. (1.8). We note that the lattice version of the equilibrium correlation func. tion is monotonously decreasing with time.

We transform the sum into an integral (using $\left.d \mathbf{q}=O_{d} q^{d-1} d q\right)$ and find in the limit $2 \pi / a \rightarrow \infty$.

$$
C_{e q}(t)=\frac{O_{d} T}{K} \int_{2 \pi / L}^{\infty} d q q^{d-3} \exp \left(-\frac{K q^{2}}{\eta} t\right) .
$$

We first consider the second moment of the scalar field by setting $t=0$. We find:

$$
\left\langle\zeta^{2}(\mathbf{r})\right\rangle \sim L^{2-d},
$$


and the limit $L \rightarrow \infty$ exists only for dimensions $d \geq 3$. We conclude that the second moment is finite for $d \geq 3$,

$$
\left\langle\zeta^{2}(\mathbf{r})\right\rangle_{e q}<\infty, \quad d \geq 3, \quad L \rightarrow \infty,
$$

while it diverges for $d=1,2$,

$$
\left\langle\zeta^{2}(\mathbf{r})\right\rangle_{e q} \rightarrow \infty, \quad d=1,2, \quad L \rightarrow \infty .
$$

In the limit $L \rightarrow \infty$, the correlation function decays algebraically in time,

$$
C_{e q}(t) \sim \frac{1}{t^{(d-2) / 2}}, \quad t \rightarrow \infty, \quad d \geq 3 .
$$

In particular, we have $C_{\text {eq }} \sim 1 / \sqrt{t}$ for $d=3$ and $C_{e q} \sim 1 / t$ for $d=4$. We note that the integral $\int_{0}^{\infty} C_{\text {eq }}(t) d t$ is finite for $d>4$, while it diverges for $d=3$. Thus $d=4$ is the critical dimension for the decay of equilibrium correlation functions. Tauberian theorems then show that the Laplace transform of the correlation function, $\hat{C}_{e q}(u)=\int_{0}^{\infty}$. $e^{-u t} C_{e q}(t) d t$, can be expanded in a Taylor series around $u=0$ for $d>4$,

$$
\hat{C}_{e q}(u)=\tau^{-1}+u+\mathcal{O}\left(u^{2}\right), \quad d>4 .
$$

Here, $\tau$ denotes an effective relaxation time for the decay of correlation functions in dimensions $d>4$.

The first and second moments (and moments of arbitrary order as well) of a Gaussian distribution are finite, and we conclude from Eq. (1.11) that the scalar field does not obey Gaussian statistics in dimensions $d=1,2$. For probability distributions other than the Gaussian, moments of the random variable do not exist to arbitrary order. For the Cauchy distribution, for example, only the first moment exists, while the second moment already diverges. Indeed, the Gaussian and Cauchy distributions are two representatives of a class of stable probability distributions. ${ }^{9-11}$ For the random variable $x$, these Lévy distributions are given by $\pi^{-1} \int_{0}^{\infty} \exp \left(-u^{D}\right) \cos (u x) d u$ with $0 \leq D \leq 2$. We recover the Gaussian distribution for $D=2$ and the Cauchy distribution for $D=1$. In his original derivation, P. Lévy was motivated by the problem of when a random walk distribution is independent of the number of steps taken. Nowadays, such random walk processes are referred to as "Lévy-flights" and "Lévy-walks". ${ }^{12}$ Lévy-flights have been introduced in statistical description of anomalous diffusion in Hamiltonian and dissipative dynamical systems. Enhanced diffusion has also been predicted in diffusion at solid-liquid interfaces. ${ }^{13}$
In this paper, we describe the stochastic processes of thermal fluctuations of the scalar field $\zeta(\mathbf{r}, t)$ in arbitrary spatial dimensions and invest $\mathrm{i}$ gate, in particular, the limit $L \rightarrow \infty$. It is only for dimensions $d>4$ that both the second moment exists and the decay of equilibrium correlation functions defines an effective relaxation time [cf. Eqs. (1.11) and (1.14)]. We conclude that for $d>4$, equilibrium fluctuations of the scalar field can be described by ordinary Brownian motion. ${ }^{14}$ For dimensions $d<4$, the long-time tailed decay of equilibrium correlation functions does not define an effective relaxation time. We expect scale-invariant properties of thermal fluctuations so that a description with ordinary Brownian motion is not possible. We show instead that a description with Lévyflights follows from principles of equilibrium statistical mechanics.

The outline of the remainder of this paper is as follows. In Sec. 2, we derive the decay of equilibrium correlation functions in one and two dimensions. Thermal fluctuations are then introduced in Sec. 3. We summarize and discuss our results in Sec. 4.

\section{DECAY OF CORRELATIONS IN ONE AND TWO DIMENSIONS}

The deterministic relaxation of the conserved scalar field, [cf. Eq. (1.2)], is the diffusion equation for the probability density of a $d$-dimensional (symmetric) random walker with $K \eta^{-1}$ being its diffusion constant. ${ }^{4}$ In particular, the probability density that a random walker starting off at the position $\mathbf{r}^{\prime}$ at time $t^{\prime}$ is at the position $\mathbf{r}$ at a later time $t>t^{\prime}$, is equal to the Green's function of the diffusion equation, $p\left(\mathbf{r}, t ; \mathbf{r}^{\prime}, t^{\prime}\right)=G\left(\mathbf{r}, t ; \mathbf{r}^{\prime}, t^{\prime}\right)$. For times $t<t^{\prime}$, the probability density vanishes. From the respective properties of the Green's function, it follows that the probability depends on the differences $r-r^{\prime}$ and $t-t^{\prime}$ only, $p\left(\mathbf{r}, t ; \mathbf{r}^{\prime}, t^{\prime}\right)=p\left(\mathbf{r}-\mathbf{r}^{\prime}, t-t^{\prime}\right)$. For a random walker on a $d$-dimensional lattice, we may define the conditional probability $p\left(\mathbf{r}_{1}, t ; \mathbf{r}_{\jmath}, t^{\prime}\right)$ as an average over its realizations. For the realization labeled $\alpha$, let $\prod_{\alpha}\left(\mathbf{r}_{i}, t ; \mathbf{r}_{j}, t^{\prime}\right)=1$ if the random walker is at the lattice point $r_{i}$ at time $t$ and $\prod_{\alpha}\left(\mathbf{r}_{i}, t ; \mathbf{r}_{j}, t^{\prime}\right)=0$ otherwise. We have:

$$
p\left(\mathbf{r}_{1}, t ; \mathbf{r}_{j}, t^{\prime}\right)=\left\langle\prod_{a}\left(\mathbf{r}_{i}, t ; \mathbf{r}_{j}, t^{\prime}\right)\right\rangle_{a} .
$$


For equal times, the condition:

$$
\prod_{\mathbf{a}}\left(\mathbf{r}_{i}, t ; \mathbf{r}_{j}, t^{\prime}\right)=\delta_{\mathbf{r}_{i} \mathbf{r}_{j}}
$$

holds. Below, we use the notation $\prod_{\alpha}\left(\mathbf{r}_{i}, t ; \mathbf{r}_{j}, t^{\prime}\right)=$ $\prod_{o}\left(\mathrm{r}_{i}-\mathrm{r}_{\jmath}, t-t^{\prime}\right)$.

We make use of a property of random walk processes first derived by Polya. ${ }^{15}$ It states that a symmetric random walker in one and two dimensions returns to its starting point with probability one. In three dimensions, the probability of return is 0.35 , and in still higher dimensions, this probability is vanishingly small. The starting point is not distinguished, and the random walker passes each lattice point with certainty in one and two dimensions. As a corollary, in one and two dimensions, each realization of the random walk returns to its origin (and passes each lattice point) an infinite number of times.

We readily find the time average of the probability density,

$$
p\left(\mathbf{r}_{i}\right)=\int_{0}^{\infty} p\left(\mathbf{r}_{i}, t\right) d t=V^{-1} \sum_{\mathbf{q}} \frac{\eta}{K q^{2}} e^{i \mathbf{q} \mathbf{r}_{\mathbf{.}}} .
$$

Upon comparison with Eq. (1.9), we conclude that the time-averaged probability density is proportional to the equilibrium correlation function,

$$
C_{e q}\left(\mathbf{r}_{i}, t=0\right)=\frac{T}{\eta} p\left(\mathbf{r}_{t}\right) .
$$

We insert Eq. (2.1) into Eq. (2.3) and interchange taking the average over realizations and the integration over time,

$$
p\left(\mathbf{r}_{t}\right)=\left\langle\int_{0}^{\infty} \prod_{\alpha}\left(\mathbf{r}_{i}, t\right) d t\right\rangle_{\alpha} .
$$

The time integral over $\prod_{a}\left(\mathbf{r}_{i}, t\right)$ is equal to the number of times the random walker is at the lattice point $\mathbf{r}_{i}$ for that realization,

$$
N_{\alpha}\left(\mathbf{r}_{i}\right)=\int_{0}^{\tau_{\infty}} \prod_{\alpha}\left(\mathbf{r}_{i}, t\right) d t, \quad \tau_{\infty} \rightarrow \infty .
$$

From Eqs. (2.4)-(2.6), we find that the second moment of the scalar field follows from properties of symmetric random walks for long times,

$$
\left\langle\zeta^{2}\left(\mathbf{r}_{i}\right)\right\rangle \sim\left\langle N_{\alpha}\left(\mathbf{r}_{i}\right)\right\rangle_{\alpha} .
$$

In dimensions $d \geq 3$, for each realization $\alpha$, the random walker does not pass the lattice point $r_{i}$ after a certain time $\tau_{a}$, i.e., $\prod_{a}\left(r_{1}, t\right)=0$ for $t>\tau_{a}$ We may choose $\tau_{\infty}>\tau_{\alpha}$ and find that $N_{\alpha}\left(\mathbf{r}_{i}\right)$ does not depend on the cut-off time $\tau_{\infty}$ for $d \geq 3$. In one and two dimensions, the random walker passes the lattice point $\mathbf{r}_{1}$ infinitely often, and $N_{\alpha}\left(\mathbf{r}_{i}\right) \rightarrow \infty$ as $\tau_{\infty} \rightarrow \infty$. Using Eq. (2.7), we recover the properties of the second moment of the scalar field that have been derived in the Introduction.

We proceed in an analogous way to derive the long-time behavior of the correlation function. Since $p\left(\mathbf{r}_{i}, t\right)=V^{-1} \sum_{\mathbf{q}} \exp \left(-t / \tau_{q}+i \mathbf{q} \cdot \mathbf{r}_{1}\right)$, we find by integration,

$$
C_{e q}\left(\mathbf{r}_{1}, t\right)=\frac{T}{\eta} \int_{0}^{\infty} p\left(\mathbf{r}_{1}, t+s\right) d s .
$$

and find,

$$
C_{e q}\left(\mathbf{r}_{i}, t\right)=\frac{T}{\eta}\left\langle\int_{0}^{\infty} \prod_{a}\left(\mathbf{r}_{i}, t+s\right) d s\right\rangle_{a} .
$$

Using the definition of $\prod_{a}\left(\mathbf{r}_{t}, t\right)$, we observe that the time integral on the RHS is equal to the number of times the random walker passes the lattice point after the arbitrary time $t$,

$$
N_{\mathbf{a}}\left(\mathbf{r}_{t}, t\right)=\int_{0}^{\tau_{\infty}} \prod_{a}\left(\mathbf{r}_{t}, t+s\right) d s, \quad \tau_{\infty} \rightarrow \infty .
$$

We have:

$$
C_{\text {eq }}\left(\mathbf{r}_{i}, t\right)=\frac{T}{\eta}\left\langle N_{\alpha}\left(\mathbf{r}_{i}, t\right)\right\rangle_{\mathbf{a}},
$$

so that the equilibrium correlation function $C_{e q}\left(\mathbf{r}_{i}, t\right)$ is determined by the average number of times the symmetric random walker returns to its starting point after the time $t$.

In dimensions $d \geq 3$, the random walker eventually goes off to infinity after a certain time $\tau_{a}$, and we have:

$$
N_{\alpha}\left(\mathbf{r}_{i}=0, t\right)=0, \quad \text { for } \quad t>\tau_{a}, \quad d \geq 3 .
$$

For a fixed time $t$, we determine the number of realizations with a nonvanishing value of $N_{\mathbf{a}}(0, t)$. This number decreases as time increases, and we conclude that $\left\langle N_{\alpha}(0, t)\right\rangle_{\alpha} \rightarrow 0$ for $t \rightarrow \infty$. We recover the decay of equilibrium correlation functions for long times,

$$
C_{e q}(t) \rightarrow 0, \quad \text { for } t \rightarrow \infty, \quad d \geq 3 .
$$

In one and two dimensions, the random walker does not go off to infinity, but rather returns to its 
starting point with probability one after the arbitrary time $t$. Moreover, the random walker returns infinitely often after the arbitrary time $t$. We conclude that the number of returns is independent of the time $t$,

$$
N_{\alpha}\left(\mathbf{r}_{i}, t\right)=N_{\alpha}\left(\mathbf{r}_{i}\right), \quad d=1,2 .
$$

For $L \rightarrow \infty$, the equilibrium correlation function does not decay in one and two dimensions,

$$
C_{e q}(t)=C_{e q}(t=0)=\text { const. } \quad d=1,2 .
$$

This behavior for $d=1,2$ complements the algebraically decaying behavior of the correlation functions for $d \geq 3$ derived in the Introduction [cf. Eq. (1.14)].

\section{EQUILIBRIUM FLUCTUATIONS}

We have assumed that the system described by the conserved scalar field $\zeta(\mathbf{r}, t)$ is in contact with a thermal reservoir at temperature $T$ [cf. Eq. (1.6)]. The combined effect of both fluctuations and dissipation drive the system towards a unique stationary state, irrespective of the state of the system at an arbitrary initial time. In this stationary state, the scalar field follows the canonical distribution $\rho_{\text {eq }}[\zeta(\mathbf{r})]=Z^{-1} \exp (-\mathcal{F}[\zeta(\mathbf{r})] / T)$, where $\mathcal{F}[\zeta(\mathbf{r})]$ is the free energy. Due to thermal fluctuations, the time evolution of the scalar field is no longer described by its deterministic relaxation equation. Rather, Eq. (1.2) must be altered to include a term describing thermal fluctuations. Both thermal fluctuations and dissipation originate from the interaction of the system with a much larger thermal reservoir. This common origin is the physical basis of the fluctuation-dissipation theorem that was first formulated by Callen and Welton. ${ }^{16}$

We briefly discuss the connection between thermal fluctuations and dissipation in systems without a conservation law. ${ }^{17}$ The deterministic relaxation equation $\eta d \zeta(t) / d t=-\zeta(t)$ is characterized by the dissipation rate $\eta^{-1}$. The stochastic time evolution follows the Langevin equation $\eta d \zeta(t) / d t=-\zeta(t)+$ $\xi(t)$, where the "fluctuating force" has a vanishing mean, $\langle\xi(t)\rangle=0$. In many cases, the stochastic process has the Gauss-Markov property and correlations between fluctuating forces at different times can be neglected, i.e., $\langle\xi(t) \xi(s)\rangle=D \delta(t-s)$. Here, $D$ is the diffusion constant which is a measure of the strength of the fluctuations. The mean square displacement of the stochastic process is then given by $\left\langle[\zeta(t)-\zeta(0)]^{2}\right\rangle=2 D t$ for $t \gg \eta$. The diffusion constant is related to the dissipation rate and temperature via the Einstein relation $D=T / \eta$.

From $\eta d \zeta(t) / d t=-\zeta(t)$, we find exponential decay $\zeta(t)=\zeta(0) \exp (-t / \eta)$. The correlation function decays exponentially in time as well, $\langle\zeta(t) \zeta(0)\rangle=\exp (-t / \eta)\left\langle\zeta^{2}(0)\right\rangle$. We conclude that correlations are negligibly small on time-scales that are longer than the relaxation time $\eta$. This property suggests the use of Gaussian "white noise" for describing thermal fluctuations. Indeed, Doob's theorem states that a Gaussian process is Markovian only when correlation functions decay exponentially in time. ${ }^{14}$

In systems with continuous symmetry, the hydrodynamic relaxation of the scalar field, $\eta \partial \zeta(\mathbf{r}, t)=$ $K \nabla^{2} \zeta(\mathbf{r}, t)$, gives an algebraic decay of its equilibrium correlation function. We find $\langle\zeta(\mathbf{r}, t) \zeta(\mathbf{r}, 0)\rangle \sim$ $t^{-(d-2) / 2}$ for $d \geq 3$ and $\langle\zeta(\mathbf{r}, t) \zeta(\mathbf{r}, 0)\rangle \sim t^{0}=$ const. for $d=1$ and $d=2$, [cf. Eq. (1.14) and (2.15)]. We now have the Langevin equation:

$$
\eta \frac{\partial \zeta(\mathbf{r}, t)}{\partial t}=K \nabla^{2} \zeta(\mathbf{r}, t)+\xi(\mathbf{r}, t)
$$

where the fluctuating force has a vanishing mean $\langle\xi(\mathbf{r}, t)\rangle_{e q}=0$. For $d \leq 3$, the decay of the correlation function does not define a finite effective relaxation time, and correlations of the fluctuating force at different times can no longer be neglected. That is, a Gauss-Markov description of the fluctuating force is no longer possible for $d \leq 3$. In Ref. 7 , Onsager's regression hypothesis has been used to find correlations of the fluctuating force. It states that correlations of fluctuating variables at times $t$ and 0 in equilibrium systems are the same as averages of fluctuating variables at time $t$, given that certain fluctuations have occurred at time $0 .{ }^{18}$ We have:

$$
\langle\xi(\mathbf{r}, t) \xi(\mathbf{r}, 0)\rangle_{e q} \sim t^{-(d-2) / 2}, \quad d \geq 3,
$$

and

$$
\langle\xi(\mathbf{r}, t) \xi(\mathbf{r}, 0)\rangle_{e q} \sim t^{0}=\text { const. }, \quad d=1,2 .
$$

We emphasize that Eqs. (3.2) and (3.3) are valid only in the scaling regime, $(4 \pi)^{2} K^{-1} \eta a^{2} \ll t \ll$ $(4 \pi)^{2} K^{-1} \eta L^{2}$.

In thermal equilibrium, the time evolution of the scalar field is given by, [cf. Eq. (3.1)]:

$$
\zeta(\mathbf{r}, t)=\eta^{-1} \int_{0}^{t} \xi(\mathbf{r}, s) d s .
$$


The mean square displacement follows as:

$$
\begin{aligned}
\sigma(t) & \equiv\left\langle[\zeta(\mathbf{r}, t)-\zeta(\mathbf{r}, 0)]^{2}\right\rangle_{e q} \\
& =2 \eta^{-2} \int_{0}^{t} d s \int_{0}^{s} d s^{\prime}\left\langle\xi(\mathbf{r}, s) \xi\left(\mathbf{r}, s^{\prime}\right)\right\rangle_{e q} .
\end{aligned}
$$

It is convenient to distinguish four different cases. First, for $d>4$, the equilibrium correlation function decays faster than the inverse of time, and its Laplace transform can be expanded in a Taylor series around the origin [cf. Eq. (1.15)]. Using theorems for Laplace transforms, we find diffusive behavior:

$$
\sigma(t) \sim \frac{t}{\tau}, \quad d>4,
$$

where $\tau$ is the effective relaxation time. We conclude that for $d>4$, the fluctuating forces are described by Gaussian white noise, i.e., $\langle\xi(\mathbf{r}, t) \xi(\mathbf{r}, s)\rangle_{e q} \sim \delta(t-s)$. For $d=4$, we have $\left\langle\xi(\mathrm{r}, s) \xi\left(\mathrm{r}, s^{\prime}\right)\right\rangle_{\text {eq }} \sim\left(s-s^{\prime}\right)^{-1}$. The mean square displacement then follows as $\sigma(t) \sim t \ln (t)$. Because $t^{-\epsilon} \ln t \rightarrow 0$ as $t \rightarrow \infty$ for $\varepsilon>0$, equilibrium fluctuations in dimensions $d>4$ and $d=4$ belong to the same universality class:

$$
\sigma(t) \sim t, \quad d=4 .
$$

For dimensions $d=3$, correlations decay more slowly, $\left\langle\xi(\mathbf{r}, s) \xi\left(\mathbf{r}, s^{\prime}\right)\right\rangle_{e q} \sim\left(s-s^{\prime}\right)^{-1 / 2}$, and we find super-diffusive behavior:

$$
\sigma(t) \sim t^{3 / 2}, \quad d=3 .
$$

Finally, for dimensions $d=1$ and $d=2$, correlations of the fluctuating forces do not decay, and we again find super-diffusive behavior:

$$
\sigma(t) \sim t^{2}, \quad d=1,2 .
$$

It is standard to summarize these findings as $\sigma(t) \sim t^{2 H}$, where $H$ is the Hurst exponent, $0<$ $H<1$. Ordinary Brownian motion is characterized by $H=\frac{1}{2}$. For $H \neq \frac{1}{2}$, Mandelbrot coined the term fractional Brownian motion. ${ }^{9}$ For $d \geq 4$, we have diffusive behavior with $H=\frac{1}{2}$, while for $d \leq 3$ we have superdiffusive behavior. In particular, we have $H=\frac{3}{4}$ for $d=3$ and $H=1$ for $d=1,2$.

Mandelbrot noted that fractional Brownian motion is a stationary stochastic process with Gaussian increments. Furthermore, these processes are continuous but non-differentiable. The corresponding spectral densities of the fluctuating forces are proportional to $f^{-(2 H-1)}$. We have the spectral densities $1 / f$ for $d=1$ and $d=2,1 / \sqrt{f}$ for $d=3$, and white noise, $f^{0}$, for $d \geq 4$. Clearly, this be havior of the spectral densities already follows from Eqs. (3.2) and (3.3).

Fractional Brownian processes yield stable distributions that depend on a continuous parameter $0 \leq D \leq 2$. For the random variable $x$, these so-called Lévy-distributions are given by $\rho_{D}(x)=$ $\pi^{-1} \int_{0}^{\infty} \exp \left(-u^{D}\right) \cos (u x) d u$. The Gaussian and Lorentzian distributions follow for $D=2$ and $D=1$, respectively. The Hurst exponent $H$ and the dimensionality $D$ are related to each other by $D=1 / H$. We have $D=2$ for $d \geq 4, D=\frac{4}{3}$ for $d=3$, and $D=1$ for $d=1$ and $d=2$. This argument gives an explicit representation of the canoni. cal distribution of the scalar field $\rho_{\text {eq }}[\zeta(\mathbf{r})]=$ $Z^{-1} \exp (-\mathcal{F}[\zeta(\mathbf{r})] / T)$, [cf. Eq. (1.6)]. We have the Gaussian distribution:

$$
\rho_{\text {eq }}[\zeta(\mathbf{r})]=\sqrt{\frac{\lambda}{\pi}} \exp \left(-\frac{\zeta^{2}(\mathbf{r})}{\lambda}\right) . \quad d \geq 4,
$$

where $\lambda=\left\langle\zeta^{2}(\mathbf{r})\right\rangle_{e q} \propto T$ and the Cauchy distribution:

$$
\rho_{\text {eq }}[\zeta(\mathbf{r})]=\frac{1}{\pi} \frac{\mu}{\zeta^{2}(\mathbf{r})+\mu^{2}}, \quad d=1,2,
$$

where $\mu \propto T$. For $d=3$, the closed expression of the stationary distribution of the scalar field is not known.

For $d \geq 4$, the characteristic length-scale is given by the second moment. $\left\langle\zeta^{2}(\mathbf{r})\right\rangle_{\text {eq }}$. Higher moments follow from the Gaussian property, e.g., $\left\langle\zeta^{4}(\mathbf{r})\right\rangle_{\text {eq }}=$ $3\left\langle\zeta^{2}(\mathbf{r})\right\rangle_{e q}^{2}=3 \lambda^{2}$. For $d=1$ and $d=2$, the second moment diverges $\left\langle\zeta^{2}(\mathbf{r})\right\rangle_{e q} \rightarrow \infty$, and the Cauchy distribution does not define a characteristic lengthscale. For $d=3$, the stationary distribution extrapolates between the Gaussian and Cauchy distribution, and moments of the scalar field do not exist to arbitrary high order.

\section{SUMMARY AND DISCUSSION}

We studied relaxation and thermal fluctuations of a conserved scalar field in arbitrary spatial dimensions. We first considered a lattice version which introduces an upper and lower frequency cut-off. $2 \pi / a$ and $2 \pi / L$, respectively. The limit $a \rightarrow 0$ is trivial, whereas taking the limit $L \rightarrow \infty$ depends on the number of spatial dimensions. We found that the second moment of the scalar field is finite only for dimensions $d>3$, while the second 
moment diverges for $d=1$ and $d=2$. Similarly, it is only for dimensions $d>4$ that the decay of equilibrium correlation functions define an effective relaxation time. These properties show that in the limit $L \rightarrow \infty$, thermal fluctuations of the scalar field lack proper length and time scales for dimensions $d<4$.

For dimensions $d>4$, proper length and time scales exist, and thus thermal fluctuations of the scalar field are described by ordinary Brownian motion. It follows, in particular, that the fluctuating force is described by Gaussian white noise. For dimensions $d<4$, thermal fluctuations of the scalar field are described by fractional Brownian motion. For the spectral densities of the fluctuating forces, we have found $1 / f$ for $d=1$ and $d=2$ and $1 / \sqrt{f}$ for $d=3$. Correspondingly, the stationary distribution of the scalar field is the Gaussian for $d>4$, while the Cauchy distribution follows for $d=1,2$.

Super-diffusive behavior has been derived from long-time tailed decay of equilibrium correlation functions and is a consequence of non-Markovian properties of the fluctuating forces. On the other hand, it is shown in Ref. 19 that Lévy-flights are exact solutions of the Bachelier-SmoluchowskyChapman-Kolmogorov equation and are essentially Markov processes. To resolve this seemingly contradictory statement, the authors of Ref. 19 show that the non-Markovian properties of Gaussian fluctuating forces become properties of transition probabilities with an inverse power-law dependence on the length of the jump in space.

In Ref. 20, an elaborate time-series analysis is used to investigate temporal fluctuations of Goldstone modes. The authors find the value $H=1$ for dimensions $d=3$, which is at variance with the result reported here. The authors fail to recognize the non-Markovian (or non-Gaussian) character of the fluctuating force that is implicit in a value of the Hurst exponent different from $\frac{1}{2}$, and instead assume the Markovian limit for the fluctuating forces. Therefore, in Ref. 20, non-local aspects of the fluctuating scalar field are not treated in a proper manner. ${ }^{19}$

In recent years, Lévy-processes have been reported in a wide variety of physical situations. This has led to the speculation that such a generalization of Brownian motion may become as ubiquitous as its predecessor. ${ }^{12}$ We have added yet another example to this ever increasing list. For a conserved scalar field, we have shown that equilibrium fluctuations are described by ordinary Brownian motion only for dimensions $d>4$, while scale-invariant properties warrant a Lévy-flight description for dimensions $d<4$.

\section{REFERENCES}

1. P. C. Hohenberg and B. I. Halperin, Rev. Mod. Phys. 49, 435 (1977).

2. S. P. Obukhov, Phys. Rev. Lett. 65, 1395 (1990).

3. X. Che and H. Suhl, Phys. Rev. Lett. 64, 1670 (1990).

4. S. F. Edwards and D. R. Wilkinson, Proc. R. Soc. A381, 17 (1982).

5. P. A. Pincus, in Structure and Dynamics of Strongly Interacting Colloids and Supramolecular Aggregates in Solution, eds. S. H. Chen, J. S. Huang and P. Tartaglia (Kluwer, Dordrecht, 1992).

6. Z. Zhang, O. G. Mouritsen, K. Otnes, T. Riste and M. J. Zuckerman, Phys. Rev. Lett. 70, 1834 (1993).

7. U. Zürcher, Phys. Rev. Lett. 72, 3367 (1994).

8. J. D. Gunton and M. San Miguel, in Phase Transitions and Critical Phenomena, Vol. 8, eds. C. Domb and J. L. Lebowitz (Academic Press, London, 1983).

9. B. B. Mandelbrot, The Fractal Geometry of Nature (Freeman, San Francisco, 1982).

10. R. N. Mantegna and H. E. Stanley, Phys. Rev. Lett. 73, 2946 (1994).

11. M. F. Shlesinger, Phys. Rev. Lett. 74, 4959 (1995) (C).

12. M. F. Shlesinger, J. Klafter and G. Zumofen, Fractals 3, 491 (1995).

13. O. V. Bychuk and B. O'Shaugnessy, Phys. Rev. Lett. 74, 1795 (1995).

14. M. C. Wang and G. E. Uhlenbeck, Rev. Mod. Phys. 17, 323 (1947); reprinted in Selected Papers on Noise and Stochastic Processes, ed. N. Wax (Dover, New York, 1954).

15. W. Feller, An Introduction to Probability Theory and Its Applications, Vols. I and II, 3rd. ed. (John Wiley, New York, 1968).

16. H. B. Callen and T. A. Welton, Phys. Rev. 83, 34 (1951).

17. See, e.g., C. W. Gardiner, Handbook of Stochastic Processes (Springer-Verlag, Berlin, 1983).

18. See, e.g., L. E. Reichl, A Modern Course in Statistical Physics (University of Texas Press, Austin, 1987).

19. G. Trefán, E. Floriani, B. J. West and P. Grigolini, Phys. Rev. E50, 2564 (1994).

20. C. Yeung, M. Rao and R. Desai, Phys. Rev. Lett. 73 , 1813 (1994). 